\title{
Lipid emulsion mitigates local anesthesia-induced central nervous system toxicity in rats
}

\author{
GANGMING WU, BIN SUN, LI LIU, JUN ZHOU, LIQUN MO, CHANGHE REN and CEHUA OU
}

Department of Anesthesiology and Pain, Affiliated Hospital of Luzhou Medical College, Luzhou, Sichuan 646000, P.R. China

Received August 12, 2014; Accepted May 6, 2015

DOI: $10.3892 /$ etm.2015.2594

\begin{abstract}
The aim of the present study was to investigate the effect of intravenously administered lipid emulsion on local anesthetic (LA)-induced central nervous system (CNS) toxicity. A total of 100 male Sprague Dawley rats were allocated at random into the following groups: Sham (A), lidocaine (B), levobupivacaine (C) and ropivacaine (D). Groups B-D were each subdivided into three subgroups: Toxic, post-conditioning and pre-conditioning. Intracerebroventricular injections of $0.9 \%$ normal saline (sham group) or LA were administered via microsyringe; in addition, a $20 \%$ lipid emulsion was injected into tail vein prior to the LA injection (pre-conditioning subgroups) or following rat respiratory arrest (post-conditioning subgroups). The heart rate, blood pressure, neurological behavior scores, neuronal density and time from LA injection to respiratory arrest, apnea and start of arrhythmia were measured. Rats in the toxic groups died due to respiratory arrest following the injection of LA into the lateral ventricle. Rats in the post-conditioning subgroups were resuscitated from the LA-induced respiratory arrest, while the pre-conditioning subgroup rats exhibited no respiratory arrest. No significant differences in heart rate were observed between the toxic and post-conditioning subgroups in the levobupivacaine and ropivacaine groups $(\mathrm{P}>0.05)$; however, a significant difference was observed between these treatment groups and the rats treated with lidocaine $(\mathrm{P}<0.01)$. A significant difference was also observed in the time from the LA injection to the onset of arrhythmia among the rats in groups $\mathrm{B}, \mathrm{C}$ and $\mathrm{D}(\mathrm{P}<0.01)$. No significant differences in the neurological behavior scores and neuronal density were observed in the hippocampal CA1 zone among group $\mathrm{C}$ and $\mathrm{D}$ rats in the post- and pre-conditioning subgroups at various time-points following treatment. Beyond that, the same phenomena regarding neurological
\end{abstract}

Correspondence to: Dr Cehua Ou, Department of Anesthesiology and Pain, Affiliated Hospital of Luzhou Medical College, 25 Taiping Street, Luzhou, Sichuan 646000, P.R. China

E-mail: oucehua1@126.com

Key words: lipid emulsion, local anesthetics, central nervous system toxicity, intracerebroventricular injection behavior scores was observed in post- and pre-conditioning subgroups of group B at 12 and $24 \mathrm{~h}$ treatment, contrasting with the statistically significant difference between post- and pre-conditioning subgroups at $6 \mathrm{~h}$ treatment $(\mathrm{P}<0.01)$. The results of the present study therefore indicate that pre- and post-conditioning with lipid emulsion effectively mitigates LA-induced CNS toxicity in rats.

\section{Introduction}

Local anesthetics (LAs) are drugs that reversibly block the transmission of nerve impulses, without affecting consciousness; however, accidental intravascular injection, drug overdose or rapid absorption from the administration site can induce severe LA systemic toxicity (LAST). LAST is a rare but potentially fatal complication of regional anesthesia, which may result in cardiovascular (CVS) and central nervous system (CNS) toxicity (1). Previously, the treatment of LAST was a challenge, as no specific therapy was available (2); however, it was later observed that a lipid emulsion was able to effectively resuscitate rats that had received an overdose of bupivacaine (3). Since then, a number of studies have reported the successful use of lipid emulsion therapy for the treatment of LAST $(4,5)$. To date, intravenous lipid emulsions have been used to treat systemic toxicity induced by a variety of LAs, including bupivacaine, levobupivacaine, ropivacaine and mepivacaine (6-8).

The resuscitative capacity of lipid emulsion for LA-induced CVS toxicity has been extensively studied (9); however, the efficacy of intravenous lipid emulsions in reducing LA-induced CNS toxicity has not been fully characterized. Oda and Ikeda (6) reported that a lipid emulsion effectively decreased the CNS and CVS toxicity induced by bupivacaine and levobupivacaine in conscious rats. Furthermore, previous clinical studies have indicated that lipid emulsion may be effective for treating LA-induced CNS toxicity $(10,11)$. The 'lipid-sink' theory suggests that lipophilic LAs are chelated by lipid emulsions in the blood, which lowers the concentration of the freebase form of the LA, thus reducing the availability of the LAs to tissues and resulting in decreased toxicity (12). The majority of previous experiments examining the effects of lipid emulsions on LAST involved the intravenous injection of LAs, followed by the intravenous administration of a lipid emulsion; therefore, it is difficult to determine whether lipid emulsions can have a direct effect on the CNS to limit toxicity. 
The aim of the present study was to evaluate the effect of intravenously administered lipid emulsions on the CNS toxicity induced by lidocaine, levobupivacaine and ropivacaine, which were directly perfused into the lateral ventricles of Sprague Dawley (SD) rats. In addition, this study aimed to compare the toxicity of these three types of LAs in a rat model of CNS toxicity.

\section{Materials and methods}

Experimental animals. In total, 100 healthy male SD rats, aged 10 weeks and weighing 240-300 g, were purchased from the Laboratory Animal Center of Luzhou Medical College (Luzhou, China) and maintained in a temperature-controlled room with a 12-h light/dark cycle and regular access to food and water. Experimental procedures were approved by the Laboratory Animal Care Committee at Luzhou Medical College. All animals received care according to the Guide for the Care and Use of Laboratory Animals (National Institutes of Health, Bethesda, MD, USA).

Animal model and experimental procedure. Rats were allocated at random into four main groups: Sham $(A ; n=10)$, lidocaine $(B, n=30)$, levobupivacaine $(C ; n=30)$ and ropivacaine ( $\mathrm{D} ; \mathrm{n}=30)$. Groups B-D were subsequently subdivided into three subgroups: Toxic, post-conditioning and pre-conditioning ( $\mathrm{n}=10$ per subgroup). The rats were weighed and subsequently anesthetized with an intraperitoneal injection of $2 \%$ sodium pentobarbital $\left(40 \mathrm{mg} \cdot \mathrm{kg}^{-1}\right)$. Heat pads (Softron, Beijing, China) were used for the maintenance of body temperature at $37^{\circ} \mathrm{C}$. Spontaneous breathing was maintained, and then a tail vein puncture, tracheal intubation and femoral artery puncture were conducted, with continuous injection of Ringer's solution (Minsheng Pharmaceutical Group Co. Ltd., Hangzhou, China) by a computerized infusion pump (Wego, Weihai, China) at a rate of $1 \mathrm{ml} \cdot \mathrm{kg}^{-1} \cdot \mathrm{min}^{-1}$. Simultaneously, electrocardiogram, heart rate and mean arterial pressure measurements of the rats were continuously recorded using a BL-420E biological function experimental recorder (Chengdu Thai Union Technology Co., Ltd., Chengdu, China). The onset of convulsions was indicated by the appearance of tonic-clonic movement in the rat legs and spike waves on an electroencephalogram, and the data were recorded. Following cerebroventricular puncture, the LA was injected gradually into the lateral ventricle using a microsyringe at a rate of $50 \mu \mathrm{l} \cdot \mathrm{min}^{-1}$. The dosages of the LAs, determined by preliminary experiments, were as follows: $0.75 \%$ lidocaine hydrochloride (200 $\mu \mathrm{l} \mathrm{kg}^{-1}$; Yashen Pharmaceutical Co. Ltd., Linzhou, China), $0.75 \%$ levobupivacaine hydrochloride (140 $\mu \mathrm{l} \cdot \mathrm{kg}^{-1}$; Hengrui Medicine Co. Ltd., Lianyungang, Jiangsu, China) and $1 \%$ ropivacaine hydrochloride $\left(200 \mu \mathrm{l} \cdot \mathrm{kg}^{-1}\right.$; Hengrui Medicine Co. Ltd.). Rats in the post-conditioning subgroups that exhibited respiratory arrest were administered $20 \%$ lipid emulsion (Intralipid; $5 \mathrm{ml} \cdot \mathrm{kg}^{-1}$; Sino-Swed Pharmaceutical Corp. Ltd., Beijing, China) via a tail vein injection, and were continually administered lipid emulsion using a computerized infusion pump at a rate of $0.25 \mathrm{ml} \cdot \mathrm{kg}^{-1} \cdot \mathrm{min}^{-1}$. Similarly, rats in the pre-conditioning subgroups received a tail vein injection of $20 \%$ lipid emulsion $\left(5 \mathrm{ml} \cdot \mathrm{kg}^{-1}\right)$, followed by infusion using a computerized infusion pump at a rate of $0.25 \mathrm{ml} \cdot \mathrm{kg}^{-1} \cdot \mathrm{min}^{-1}$; however, this treatment was administered for $30 \mathrm{~min}$ prior to the intracerebroventricular injection of LA. Sham group rats received $0.9 \%$ normal saline $\left(2 \mu \mathrm{l} \cdot \mathrm{kg}^{-1}\right)$, which was injected into the lateral ventricle at a rate of $50 \mu \mathrm{l} \cdot \mathrm{min}^{-1}$. The time between the injection of LA and the onset of respiratory arrest and arrhythmia was recorded in all groups. Neurological behavior was evaluated using a previously described method $(13,14)$, and the resulting scores were recorded prior to the LA treatment and at 6, 12 and $24 \mathrm{~h}$ after treatment. At $24 \mathrm{~h}$ after treatment, the rats were sacrificed. Firstly, the rats were anaesthetized by $2 \%$ pentobarbital intraperitoneal injection, and then $4 \%$ paraformaldehyde was perfused rapidly, with the aim of reducing animal suffering as much as possible. The brains were excised, fixed in $4 \%$ paraformaldehyde and embedded in paraffin blocks. Subsequently, 4- $\mu$ m sections were stained with hematoxylin and eosin using standard protocols. Images were obtained using a Nikon Eclipse E400 optical microscope and software package (Nikon Corp., Tokyo, Japan), and neuronal density in the hippocampal CA1 zone was subsequently measured. Cones cell morphology of hippocampal CA1 was observed under the microscope, with the same view captured under magnification $\mathrm{x} 100$ and $\mathrm{x} 400$. The cell morphological differences between groups were observed and neuronal density was measured.

Statistical analysis. Results are expressed as the mean \pm standard deviation. Statistical analysis was performed using SPSS software, version 17.0 (SPSS, Inc., Chicago, IL, USA). Differences in the heart rate, blood pressure, neurological behavior scores, neuronal density and time from LA injection to respiratory arrest, apnea and start of arrhythmia among the groups were examined using one-way analysis of variance. $\mathrm{P}<0.05$ was considered to indicate a statistically significant difference.

\section{Results}

Lipid emulsion mitigates LA-induced respiratory arrest. Prior to treatment, no differences in baseline weight, blood pressure, heart rate and respiration were observed among the groups (data not shown). The lateral ventricle was punctured successfully in all rats, and $0.9 \%$ normal saline or the respective LAs were injected. LAST onset was observed in all rats following the injection of LA into the lateral ventricle. Rats in the toxic subgroups all died from respiratory arrest. Rats in the post-conditioning subgroups also exhibited respiratory arrest; however, following the application of cardiopulmonary resuscitation (via closed chest cardiac massage) and breathing support (assisted with a ventilator), all rats were resuscitated from the CNS toxicity-induced respiratory arrest. No respiratory arrest was observed in the rats in the pre-conditioning subgroups.

Effect of pre-and post-conditioning on time to respiratory arrest and apnea. Following the intracerebroventricular injection of LA, the time to respiratory arrest was compared among the toxic subgroups. The data showed no significant differences between the toxic subgroups of groups B and C $(\mathrm{P}>0.05)$; however, these subgroups differed significantly compared with the group D toxic subgroup ( $\mathrm{P}<0.01$; Fig. 1A). 

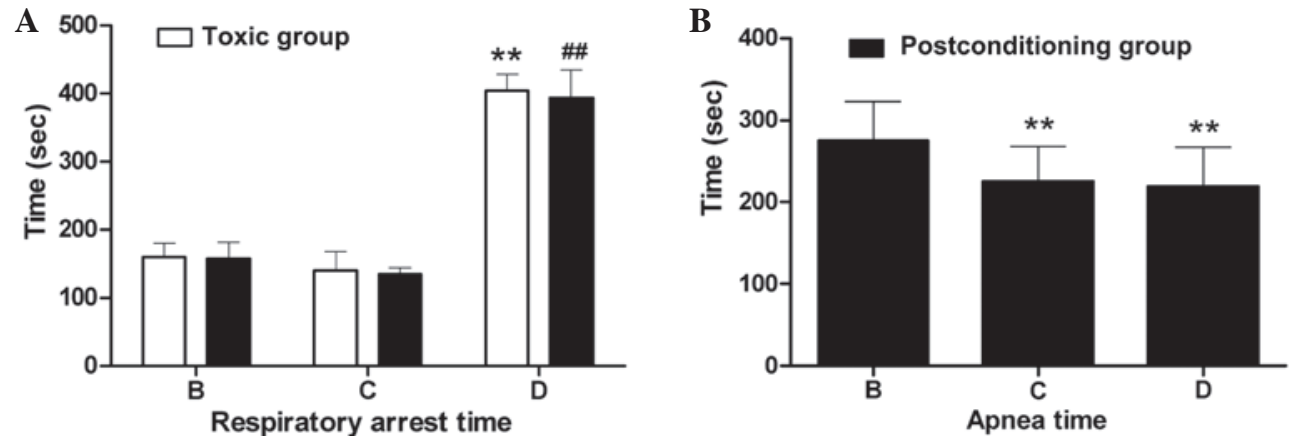

Figure 1. Time to (A) respiratory arrest and (B) apnea in the toxic and post-conditioning groups. Data are expressed as the mean \pm standard deviation. (A) ${ }^{* *} \mathrm{P}<0.01$ vs. groups $\mathrm{B}$ and $\mathrm{C}$ (toxic subgroups); ${ }^{\# \#} \mathrm{P}<0.01$ vs. groups $\mathrm{B}$ and $\mathrm{C}$ (post-conditioning subgroups). (B) ${ }^{* *} \mathrm{P}<0.01$ vs. group $\mathrm{B}$ (post-conditioning subgroup). Group B, lidocaine group; group C, levobupivacaine group; group D, ropivacaine group.
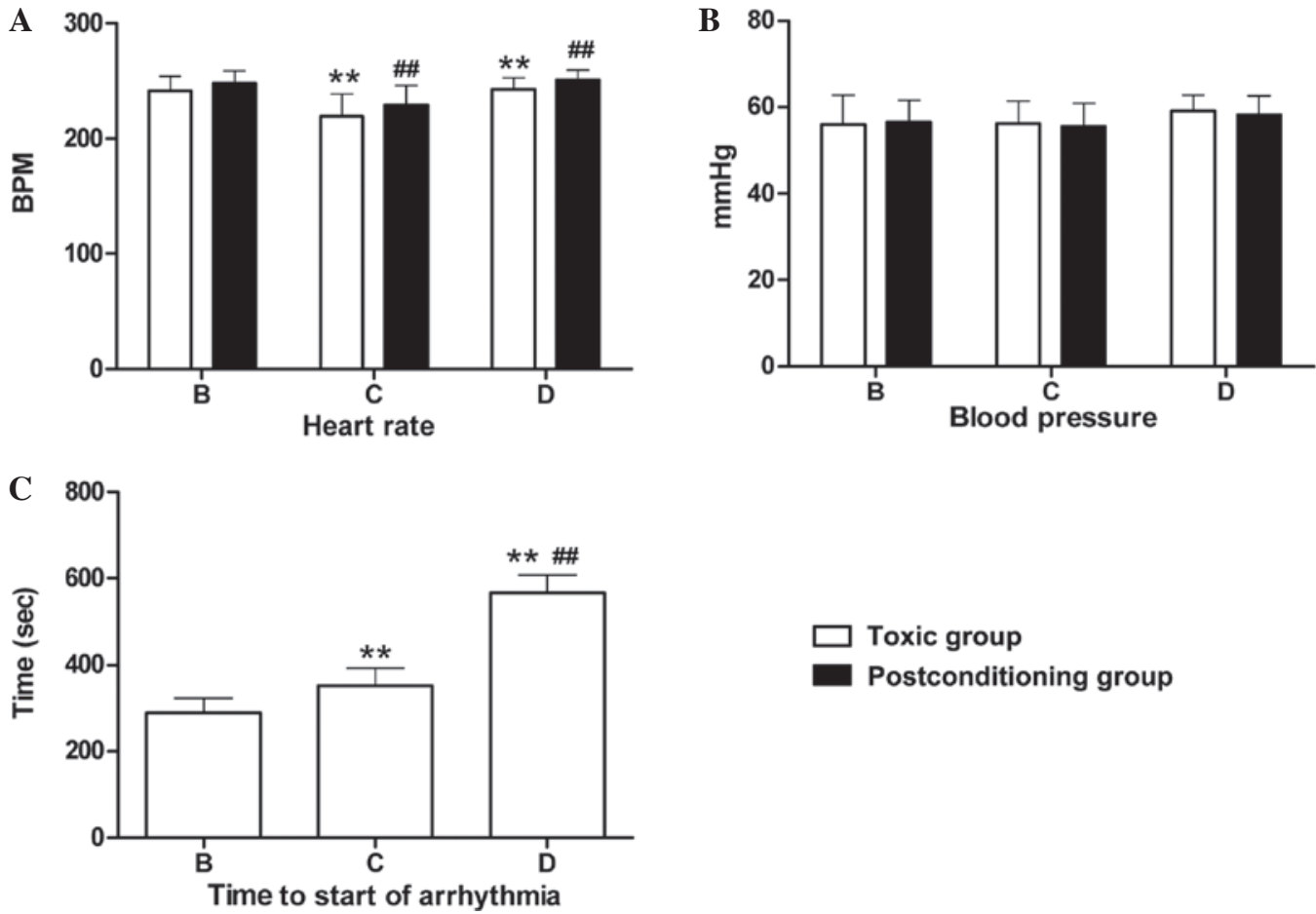

Figure 2. (A) Heart rate, (B) blood pressure and (C) time to start of arrhythmia in the toxic and post-conditioning subgroups. Data are expressed as the mean \pm standard deviation. (A) ${ }^{* *} \mathrm{P}<0.01$ vs. group B (toxic subgroup); ${ }^{\#} \mathrm{P}<0.01$ vs. group B (post-conditioning subgroup). $(\mathrm{C}){ }^{* *} \mathrm{P}<0.01 \mathrm{vs}$. group $\mathrm{B}$; ${ }^{\# \#} \mathrm{P}<0.01$ vs. group C (toxic subgroup). BPM, beats per minute; group B, lidocaine group; group C, levobupivacaine group; group D, ropivacaine group.

Similarly, no significant differences were detected between the post-conditioning subgroups of groups $\mathrm{B}$ and $\mathrm{C}(\mathrm{P}>0.05)$, while significant differences were observed between these subgroups and the group D post-conditioning subgroup $(\mathrm{P}<0.01$; Fig. 1A). Within groups $\mathrm{B}, \mathrm{C}$ and $\mathrm{D}$, no significant differences were observed in respiratory arrest time between the respective toxic and post-conditioning subgroups $(\mathrm{P}>0.05$; Fig. 1A). No respiratory arrest was exhibited by rats in the pre-conditioning subgroup rats of groups B-D. With regard to apnea time, significant differences were detected among the post-conditioning subgroups of groups $\mathrm{B}, \mathrm{C}$ and $\mathrm{D}(\mathrm{P}<0.01$; Fig. 1B). The decreasing order of recovery time was as follows: Lidocaine (group B) > levobupivacaine (group C) > ropivacaine (group D).

Heart rate, blood pressure and arrhythmia. Heart rates were measured when respiratory arrest occurred in the toxic and post-conditioning subgroups following the intracerebroventricular injection of LA. No significant difference in heart rate was observed between the toxic subgroups of groups $C$ and $\mathrm{D}(\mathrm{P}>0.05)$; however, significant differences were detected between these subgroups and the toxic subgroup of group B ( $<<0.01$; Fig. 2A). Similar results were observed among the post-conditioning subgroups of groups B, C and D. Within groups B-D, there were no significant differences in heart rate between the respective toxic and post-conditioning subgroups ( $\mathrm{P}>0.05$; Fig. 2A). Similarly, no significant differences in blood pressure were detected when respiratory arrest occurred among the respective toxic and post-conditioning subgroups following the intracerebroventricular injection of the LAs (Fig. 2B). Arrhythmia was detected only in the toxic subgroups of groups B, C and D. The types of arrhythmia detected included bradycardia, premature contraction and irregular heartbeats. Significant differences in the time 
Table I. Neurological behavior scores at various time-points (mean \pm standard deviation).

\begin{tabular}{|c|c|c|c|c|}
\hline Group & Baseline & $6 \mathrm{~h}$ & $12 \mathrm{~h}$ & $24 \mathrm{~h}$ \\
\hline Sham (A) & $17.50 \pm 1.27$ & $12.70 \pm 0.95^{\mathrm{b}-\mathrm{g}}$ & $14.90 \pm 0.99^{\mathrm{b}-\mathrm{g}}$ & $15.90 \pm 0.74^{\mathrm{b}-\mathrm{g}}$ \\
\hline \multicolumn{5}{|c|}{ Lidocaine (B) } \\
\hline 1 & $16.40 \pm 1.07$ & 0 & 0 & 0 \\
\hline 2 & $16.60 \pm 0.84$ & $9.00 \pm 0.67^{\mathrm{a}, \mathrm{c}-\mathrm{e}}$ & $9.40 \pm 0.70^{\mathrm{a}}$ & $12.40 \pm 1.17^{\mathrm{a}}$ \\
\hline 3 & $16.70 \pm 0.95$ & $10.0 \pm 1.05^{\mathrm{a}, \mathrm{b}, \mathrm{d}, \mathrm{e}}$ & $10.09 \pm 1.45^{\mathrm{a}}$ & $12.80 \pm 1.39^{\mathrm{a}}$ \\
\hline \multicolumn{5}{|c|}{ Levobupivacaine (C) } \\
\hline 1 & $16.10 \pm 1.20$ & 0 & 0 & 0 \\
\hline 2 & $15.30 \pm 0.95$ & $8.10 \pm 0.32^{\mathrm{a}-\mathrm{c}, \mathrm{f}, \mathrm{g}}$ & $9.10 \pm 0.57^{\mathrm{a}}$ & $11.70 \pm 0.95^{\mathrm{a}, \mathrm{g}}$ \\
\hline 3 & $15.80 \pm 0.92$ & $7.90 \pm 0.88^{\mathrm{a}-\mathrm{c}, \mathrm{f,g}}$ & $9.20 \pm 0.63^{\mathrm{a}}$ & $11.50 \pm 0.85^{\mathrm{a}, \mathrm{g}}$ \\
\hline \multicolumn{5}{|c|}{ Ropivacaine (D) } \\
\hline 1 & $15.80 \pm 1.03$ & 0 & 0 & 0 \\
\hline 2 & $16.10 \pm 0.88$ & $8.80 \pm 0.63^{\mathrm{a}, \mathrm{d}, \mathrm{e}}$ & $9.00 \pm 0.47^{\mathrm{a}}$ & $11.90 \pm 1.10^{\mathrm{a}}$ \\
\hline 3 & $16.30 \pm 1.16$ & $9.00 \pm 0.67^{\mathrm{a}, \mathrm{d}, \mathrm{e}}$ & $9.70 \pm 0.67^{\mathrm{a}}$ & $12.30 \pm 0.67^{\mathrm{a}}$ \\
\hline
\end{tabular}

${ }^{\text {a }} \mathrm{P}<0.01$ vs. group $\mathrm{A} ;{ }^{\mathrm{b}} \mathrm{P}<0.01$ vs. group $\mathrm{B} 2$; ${ }^{\mathrm{C}} \mathrm{P}<0.01$ vs. group $\mathrm{B} 3$; ${ }^{\mathrm{d}} \mathrm{P}<0.01$ vs. group $\mathrm{C} 2$; ${ }^{\mathrm{e}} \mathrm{P}<0.01$ vs. group $\mathrm{C} 3$, ${ }^{\text {f }} \mathrm{P}<0.01$ vs. group $\mathrm{D} 2$; ${ }^{\text {g }} \mathrm{P}<0.01$ vs. group D3. 1, toxic subgroup; 2, post-conditioning subgroup; 3, pre-conditioning subgroup.

A
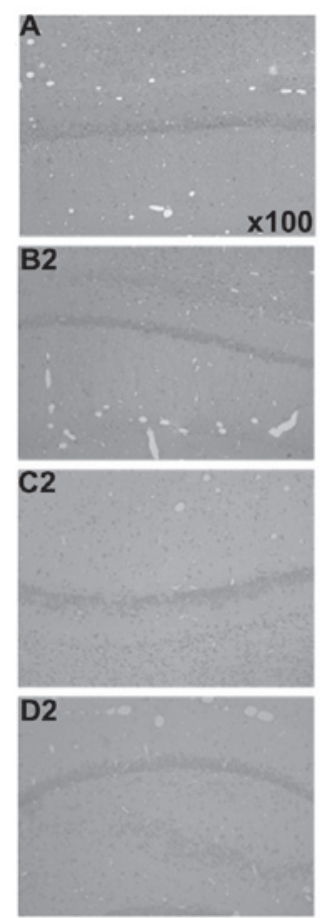
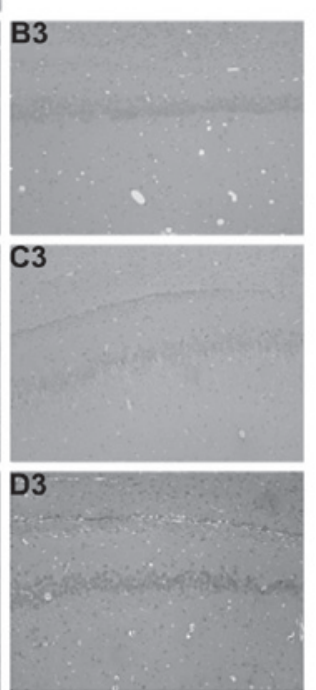

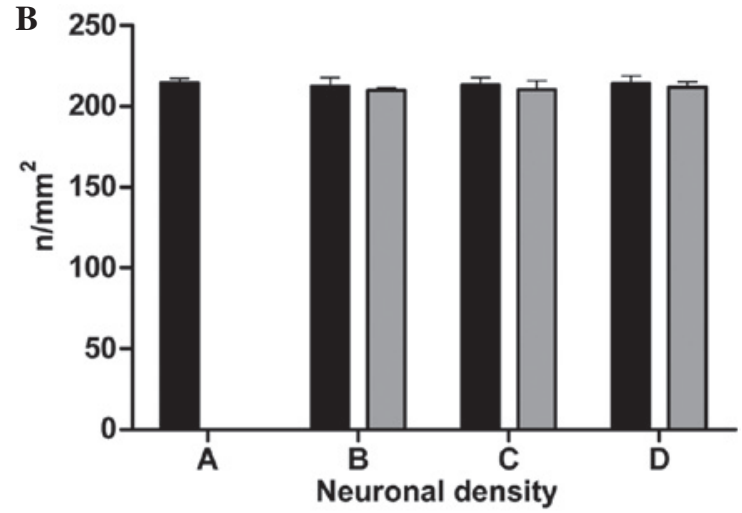

Postconditioning group Preconditioning group

Figure 3. Neuronal density in the hippocampal CA1 zones of the post- and pre-conditioning subgroups. (A) Representative images of the hematoxylin and eosin-stained rat hippocampal CA1 zone (magnification, x100). Images labeled '2' represent the post-conditioning subgroups; images labeled ' 3 ' represent the pre-conditioning subgroups. (B) Quantitative data of neuronal density in the hippocampal CA1 zone. Data are expressed as the mean \pm standard deviation. $\mathrm{n} / \mathrm{mm}^{2}$, neurons per $\mathrm{mm}^{2}$; Group A, sham group; group B, lidocaine group; group C, levobupivacaine group; group D, ropivacaine group.

from LA injection to arrhythmia onset were detected among groups $\mathrm{B}, \mathrm{C}$ and $\mathrm{D}(\mathrm{P}<0.01)$. The decreasing order of onset time was as follows: Ropivacaine (group D) $>$ levobupivacaine (group C) > lidocaine (group B) (Fig. 2C).

Neurobehavioral scores and neuronal density. Prior to LA injection, neurological behavior scores were normal in all groups. Following treatment, all the rats in the toxic subgroups died. The neurological scores of rats in the post- and pre-conditioning subgroups of groups B, C and D were significantly reduced compared with the scores of the sham group rats at the corresponding time-points following treatment $(\mathrm{P}<0.01)$. The neurological behavior of groups B, C and D treated after 6, 12 and $24 \mathrm{~h}$ was significantly lower than the sham group $(\mathrm{P}<0.01)$. There was no statistically significant difference between pre- and post-conditioning subgroups of groups B, C and D 
at different time-points, respectively $(\mathrm{P}>0.05)$. Differences were statistically significant in the sham group at 6,12 and $24 \mathrm{~h}$ time-points; toxic group, pre- and post-conditioning subgroups are significantly different from rat models treated for $24 \mathrm{~h}(\mathrm{P}<0.01$; Table I). Results for the neuronal density in the rat hippocampal CA1 zones are presented in Fig. 3A and B. The results showed that the neuronal hippocampal density was normal in each group at $24 \mathrm{~h}$ after treatment, with no significant differences observed among the various treatment groups $(\mathrm{P}>0.05)$.

\section{Discussion}

Previous animal studies and human case reports have provided increasing evidence in support of the use of lipid emulsions for the treatment of patients suffering from LA-induced toxicity $(3-5,15,16)$. Weinberg et al (3) demonstrated that LA-induced cardiotoxicity could be treated effectively using 10, 20 and 30\% lipid emulsions. Animal studies using rats and dogs determined that the use of lipid emulsion as a pretreatment or during resuscitation offered successful recovery from bupivacaine overdose $(15,17)$. Previous studies have predominantly focused on the effective resuscitative effect of lipid emulsion on LA-induced CVS toxicity (9); however, the capacity of intravenous lipid emulsions to effectively reduce LA-induced CNS toxicity has remained incompletely understood. Furthermore, based on the lipid-sink theory, the combined intravenous injection of LAs and lipid emulsion has made it difficult to determine whether lipid emulsions can have a direct effect on the CNS to prevent toxicity (12).

In the present study, an SD rat model was employed in which LAs were directly perfused into the lateral ventricle. The LAs were thus able to cross the blood-brain barrier and perfuse into the ventricle, inducing CNS, but not CVS, toxicity. This model facilitated the specific examination of the effect of lipid emulsions on LA-induced CNS toxicity. The results showed that rats in the toxic subgroups all died from breathing arrest following the injection of LA into the lateral ventricle. However, in rats treated with lipid emulsion following LA injection, respiration was restored and all rats were resuscitated from CNS toxicity-induced respiratory arrest. Rats pretreated with lipid emulsion prior to the injection of LA exhibited no respiratory arrest. Further experiments revealed no significant differences in heart rate or blood pressure following respiratory arrest between the toxic and post-conditioning subgroups. In addition, no significant differences were detected in neurological behavior scores and neuronal density in the hippocampal CA1 zone among the post- and pre-conditioning subgroups of groups B, C and D at different points following treatment. Collectively, these results suggest that post-conditioning with lipid emulsion is able to mitigate the effects of CNS toxicity, while pre-conditioning is able to prevent LA-induced CNS toxicity. To the best of our knowledge, the present study is the first to examine the resuscitative effects of lipid emulsion on LA-induced CNS toxicity.

In contrast to numerous clinical reports describing the effect of lipid emulsion on the overdose of various LAs, such as bupivacaine, levobupivacaine, ropivacaine and lidocaine $(4,5,10,18)$, the majority of animal studies have investigated bupivacaine. Few studies have examined the effects of lipid emulsion on the toxicity of LAs other than bupivacaine in animals (19). In the present study, the toxicity of three types of LA, lidocaine, levobupivacaine and ropivacaine, was examined and compared. The data showed that lipid emulsion was able to decrease the CNS toxicity induced by all three LAs, but that the toxicity of the three LAs differed. The relative respiratory recovery time observed for each of the LAs was as follows, in decreasing order: Lidocaine $>$ levobupivacaine $>$ ropivacaine. Furthermore, in decreasing order, the time from the LA injection to the onset of arrhythmia was as follows: Ropivacaine $>$ levobupivacaine $>$ lidocaine. These results suggest that the relative toxicity of the three LAs is as follows: Lidocaine $>$ levobupivacaine $>$ ropivacaine. The lipophilicity of these LAs is known to be as follows: Lidocaine $>$ levobupivacaine $>$ ropivacaine $(16,20-23)$. Notably, the apparent toxicity of these three LAs correlates with their lipophilicity, which is consistent with the lipid-sink theory.

In conclusion, the present study suggests that lipid emulsion post-conditioning is able to mitigate LA-induced CNS toxicity, while pre-conditioning is able to prevent LA-induced CNS toxicity in rats. Further studies are required to elucidate the mechanism underlying the interaction between lipid emulsions and LAs in CNS toxicity.

\section{Acknowledgements}

This study was supported by grants from the Medical Scientific Research Foundation of Sichuan Province (China), Health and Family Planning Commission of Sichuan Province (no. 110334), the Affiliated Hospital of Luzhou Medical College of Sichuan Province (no. 11208). The authors would like to thank Dr Changhe Ren and Master Liqun Mo for help with experimental methods and Dr Jun Zhou for the data analysis.

\section{References}

1. Wolfe JW and Butterworth JF: Local anesthetic systemic toxicity: Update on mechanisms and treatment. Curr Opin Anaesthesiol 24: 561-566, 2011.

2. Zink W and Graf BM: Toxicology of local anesthetics. Clinical, therapeutic and pathological mechanisms. Anaesthesist 52: 1102-1123, 2003 (In German.)

3. Weinberg GL, VadeBoncouerT, Ramaraju GA, Garcia-Amaro MF and Cwik MJ: Pretreatment or resuscitation with a lipid infusion shifts the dose-response to bupivacaine-induced asystole in rats. Anesthesiology 88: 1071-1075, 1998.

4. Rosenblatt MA, Abel M, Fischer GW, Itzkovich CJ and Eisenkraft JB: Successful use of a $20 \%$ lipid emulsion to resuscitate a patient after a presumed bupivacaine-related cardiac arrest. Anesthesiology 105: 217-218, 2006.

5. Litz RJ, Popp M, Stehr SN and Koch T: Successful resuscitation of a patient with ropivacaine-induced asystole after axillary plexus block using lipid infusion. Anaesthesia 61: 800-801, 2006.

6. Oda Y and Ikeda Y: Effect of lipid emulsion on the central nervous system and cardiac toxicity of bupivacaine and levobupivacaine in awake rats. J Anesth 27: 500-504, 2013.

7. Sakai T, Manabe W, Kamitani T, Takeyama E and Nakano S: Ropivacaine-induced late-onset systemic toxicity after transversus abdominis plane block under general anesthesia: Successful reversal with $20 \%$ lipid emulsion. Masui 59: 1502-1505, 2010 (In Japanese).

8. Dix SK, Rosner GF, Nayar M, et al: Intractable cardiac arrest due to lidocaine toxicity successfully resuscitated with lipid emulsion. Crit Care Med 39: 872-874, 2011.

9. Weinberg GL: Treatment of local anesthetic systemic toxicity (LAST). Reg Anesth Pain Med 35: 188-193, 2010. 
10. Foxall G, McCahon R, Lamb J, Hardman JG and Bedforth NM: Levobupivacaine-induced seizures and cardiovascular collapse treated with Intralipid. Anaesthesia 62: 516-518, 2007.

11. Lange DB, Schwartz D, DaRoza G and Gair R: Use of intravenous lipid emulsion to reverse central nervous system toxicity of an iatrogenic local anesthetic overdose in a patient on peritoneal dialysis. Ann Pharmacother 46: e37, 2012.

12. Weinberg G, Lin B, Zheng S, et al: Partitioning effect in lipid resuscitation: Further evidence for the lipid sink. Crit Care Med 38: 2268-2269, 2010.

13. Dong WK, Bledsoe SW, Eng DY, et al: Profound arterial hypotension in dogs: Brain electrical activity and organ integrity. Anesthesiology 58: 61-71, 1983.

14. Dong WK, Bledsoe SW, Chadwick HS, Shaw CM and Hornbein TF: Electrical correlates of brain injury resulting from severe hypotension and hemodilution in monkeys. Anesthesiology 65: 617-625, 1986.

15. Weinberg G, Ripper R, Feinstein DL and Hoffman W: Lipid emulsion infusion rescues dogs from bupivacaine-induced cardiac toxicity. Reg Anesth Pain Med 28: 198-202, 2003.

16. Mulroy MF: Systemic toxicity and cardiotoxicity from local anesthetics: Incidence and preventive measures. Reg Anesth Pain Med 27: 556-561, 2002.
17. Aumeier, C, Kasdor B, Gruber M, et al: Lipid emulsion pretreatment has different effects on mepivacaine and bupivacaine cardiac toxicity in an isolated rat heart model. Br J Anaesth 112: 735-741, 2014

18. Litz RJ, Roessel T, Heller AR and Stehr SN: Reversal of central nervous system and cardiac toxicity after local anesthetic intoxication by lipid emulsion injection. Anesth Analg 106: 1575-1577, table of contents, 2008.

19. Ok SH, Sohn JT, Baik JS, et al: Lipid emulsion reverses Levobupivacaine-induced responses in isolated rat aortic vessels. Anesthesiology 114: 293-301,2011.

20. Mather LE, Copeland SE and Ladd LA: Acute toxicity of 1ocal anesthetics: Underlying pharmacokinetic and pharmacodynamic concepts. Reg Anesth Pain Med 30, 553-566, 2005.

21. Groban L: Central nervous system and cardiac effects from longacting amide local anesthetic toxicity in the intact animal model. Reg Anesth Pain Med 28: 3-11, 2003.

22. Chang DHT, Ladd LA, Copeland S, et al: Direct cardiac effects of intracoronary bupivacaine/levobupivacaine and ropivacaine in the sheep. Br J Pharmacology 132, 649-658, 2001.

23. Casati A, Chelly JE, Cerchierini E, et al: Clinical properties of levobupivacaine or racemic bupivacaine for sciatic nerve block. J Clin Anesth 14, 111-114, 2002. 